\title{
A bridge to complex disease
}

The positional cloning of a human disease gene is a landmark event. Good examples are provided by Bardet-Biedl syndrome (BBS), for which several genes have recently been identified (see Highlights, July). But these landmarks have now assumed greater significance, because some cases of BBS seem to require at least three mutant alleles. As a clear-cut example of 'triallelic' inheritance, BBS might therefore provide some general lessons about the interaction between genes, and its influence on phenotype.

BBS is usually thought of as an autosomalrecessive disorder that is characterized by a broad and variable phenotype, involving retinal dystrophy, obesity, mental retardation, renal defects and polydactyly. BBS is also genetically heterogeneous - defects at several loci can cause the disease. So, when Katsanis et al. screened patients for mutations in two known BBS genes (BBS2 and BBS6), it was no surprise to find mutations in these genes in only some of the patients. What was more surprising was that some affected individuals

\section{GENOME EVOLUTION}

\section{A primate example of positive selection}

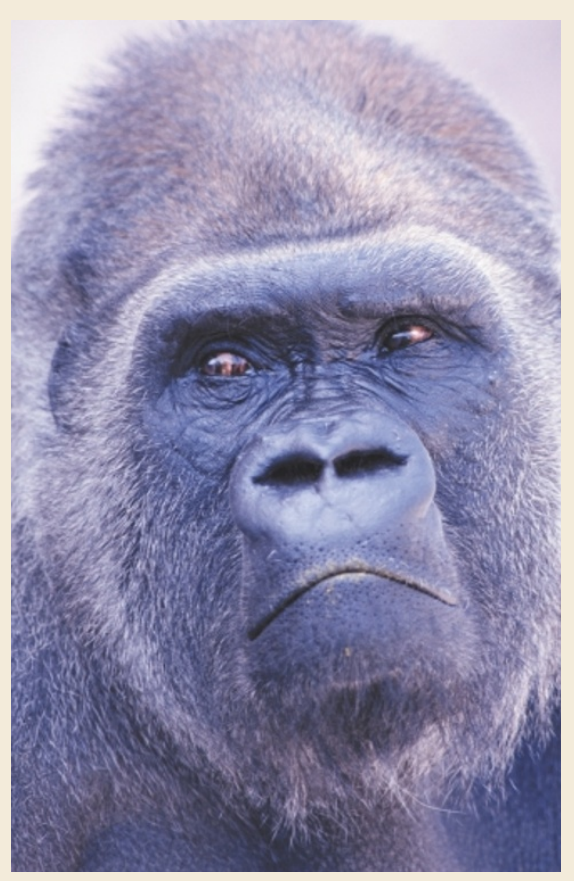

carried three mutant alleles - for example, two in BBS2 and one in BBS6.

The authors propose that BBS is inherited, at least in some individuals, in a triallelic fashion, although further BBS genes will need to be identified to determine the generality of this observation. In an accompanying Perspective, Arthur Burghes and colleagues, describe BBS inheritance as autosomal recessive with a modifier of penetrance, and liken BBS to other disorders in which modifier loci have been identified. However these unexpected findings are described, BBS provides a clear example of a phenotype that is determined by interactions between a small number of genes. BBS inheritance therefore lies somewhere between Mendelian and polygenic. As researchers move towards a more molecular understanding of BBS pathology, this disorder will become a valuable source of information about potential genetic interactions in more complex genetic disease.

Mark Patterson

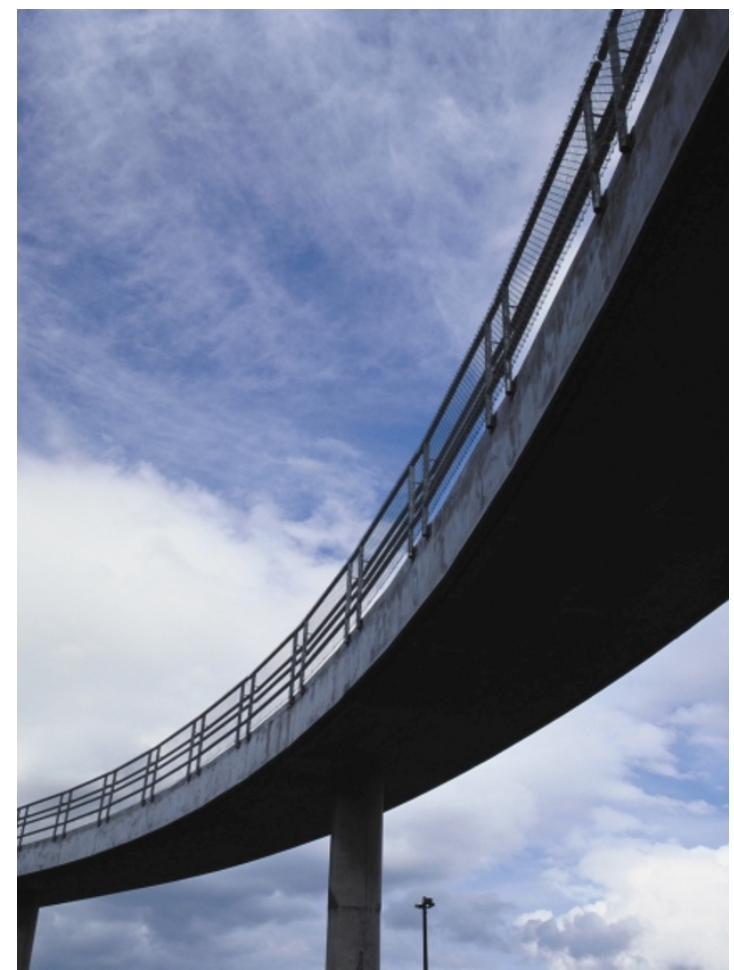

(2) References and links

ORIGINAL RESEARCH PAPER Katsanis, N. et al. Triallelic inheritance in Bardet-Biedl syndrome, a Mendelian recessive disorder. Science 293, 2256-2259 (2001)

FURTHER READING Burghes, A. H. M. et al. The land between Mendelian and multifactorial inheritance. Science $\mathbf{2 9 3}$, 2213-2214 (2001) | Nadeau, J. H. Modifier genes in mice and humans. Nature Rev. Genet. 2, 165-174 (2001) WEB SITES

James Lupski's lab: http://imgen.bcm.tmc.edu/molgen/lupsk Bardet-Biedl syndrome links:

http://www.kumc.edu/gec/support/laurmoon.html
Segmental duplications are a common feature of the human genome and they can be very difficult to sequence. But they should not be ignored. Through careful analysis of one set of segmental duplications, Matthew Johnson and colleagues have discovered a new human gene family. Furthermore, this gene family seems to have been subjected to powerful positive selection.

The authors focused on a $20-\mathrm{kb}$ repeated segment that is confined to a $15-\mathrm{Mb}$ region of chromosome 16. There are 15 copies of this segment, which have very high levels of sequence similarity. To study the evolutionary history of the duplicated segments, the authors identified the orthologous sequences in a series of primates. Their analysis showed that the segment is only present in one or two copies in Old World monkeys, such as the baboon. By contrast, in great apes such as gorillas, which are more closely related to humans, there are 9-30 copies of the segment. Overall, the segment seems to have been duplicated recently and independently in several primate lineages, after the divergence of humans and great apes from Old World monkeys.

When the sequence of the human segment was used in a database search, the authors found that an expressed sequence lurks within the repeated segment, although no homologues could be found in other organisms.
Surprisingly, sequence comparisons of the human repeats showed that the putative protein-coding regions are five times more divergent compared with the non-coding regions of the repeat. This indicates that the gene might be under positive selection for adaptive mutations. In support of this, the ratio of nonsynomous to synonymous amino-acid changes was significantly greater than 1 , and on the basis of comparisons with the primate sequences, evidence of positive selection could be found during the divergence of the great ape and human lineages. Despite the relatively recent duplication events, the gene has undergone major evolutionary change. Positive selection for amino-acid substitutions indicates an important function, which, for this gene family, is as yet unknown. Nevertheless, these observations attest to the importance of duplication and divergence as key evolutionary mechanisms. So, although segmental duplications can be a positive nuisance for genome sequencers, they provide some fascinating clues about the history of our genome.

Mark Patterson

(2) References and links ORIGINAL RESEARCH PAPER Johnson, M. E. et al. Positive selection of a gene family during the emergence of humans and African apes. Nature 413, 514-519 (2001) WEB SITE

Evan Eichler's lab: http://genetics.gene.cwru.edu/eichler/ 